\title{
PARTIAL EXACT CONTROLLABILITY \\ AND EXPONENTIAL STABILITY IN HIGHER-DIMENSIONAL LINEAR THERMOELASTICITY
}

\author{
WEIJIU LIU
}

\begin{abstract}
The problem of partial exact boundary controllability and exponential stability for the higher-dimensional linear system of thermoelasticity is considered. By introducing a velocity feedback on part of the boundary of the thermoelastic body, which is clamped along the rest of its boundary, to increase the loss of energy, we prove that the energy in the system of thermoelasticity decays to zero exponentially. We also give a positive answer to a related open question raised by Alabau and Komornik for the Lamé system. Via Russell's "Controllability via Stabilizability" principle, we then prove that the thermoelastic system is partially controllable with boundary controls without smallness restrictions on the coupling parameters.
\end{abstract}

\section{INTRODUCTION}

Let $\Omega$ be a bounded domain in $\mathbb{R}^{n}$ with smooth boundary $\Gamma=\partial \Omega$ of class $C^{2}$, and consider a $n$-dimensional linear, homogeneous, isotropic, and thermoelastic body occupying $\Omega$ in its non-deformed state. For a material point with configuration $x=\left(x_{1}, \cdots, x_{n}\right)$ at time $t$, let $u(x, t)=\left(u_{1}(x, t), \cdots\right.$, $\left.u_{n}(x, t)\right)$ and $\theta(x, t)$ denote the displacement and temperature deviation, respectively, from the natural state of the reference configuration. Then $u$ and $\theta$ satisfy the system of thermoelastic equations

$$
\begin{cases}u^{\prime \prime}-\mu \Delta u-(\lambda+\mu) \nabla \operatorname{div} u+\alpha \nabla \theta=0 & \text { in } \Omega \times(0, \infty), \\ \theta^{\prime}-\Delta \theta+\beta \operatorname{div} u^{\prime}=0 & \text { in } \Omega \times(0, \infty), \\ u(0)=u^{0}, u^{\prime}(0)=u^{1}, \theta(0)=\theta^{0} & \text { in } \Omega,\end{cases}
$$

in the absence of external forces and heat sources, where $\lambda, \mu>0$ are Lamés constants and $\alpha, \beta>0$ the coupling parameters. By 'we denote the derivative with respect to the time variable. $\Delta, \nabla$, div denote the Laplace, gradient, and divergence operators in the space variables, respectively. $u(0)$, $u^{\prime}(0)$ and $\theta(0)$ denote the functions $x \rightarrow u(x, 0), x \rightarrow u^{\prime}(x, 0)$ and $x \rightarrow$ $\theta(x, 0)$, respectively. For the derivation of (1.1), we refer to [17] and [40].

The goal of this paper is to study the exponential stability and partial exact controllability of system (1.1). Our main results will be presented in Section 2 and proved in Section 3.

School of Mathematics and Applied Statistics, University of Wollongong, Northfields Avenue, Wollongong, NSW 2522, Australia. E-mail: weijiu_liu@uow.edu.au

Submitted April 15, 1997. Revised October 13, 1997. Accepted for publication December 10, 1997.

(c) Société de Mathématiques Appliquées et Industrielles. Typeset by TEX. 
Before we give our main results, let us briefly describe the existing literature.

Under the Dirichlet-Dirichlet boundary conditions

$$
u=0, \quad \theta=0 \quad \text { on } \Gamma \times(0, \infty)
$$

the thermoelastic energy of (1.1) can be defined as

$$
\begin{aligned}
E(t)= & \frac{1}{2} \int_{\Omega}\left[\left|u^{\prime}(x, t)\right|^{2}+\mu|\nabla u(x, t)|^{2}\right. \\
& \left.+(\lambda+\mu)|\operatorname{div} u(x, t)|^{2}+\frac{\alpha}{\beta}|\theta(x, t)|^{2}\right] d x .
\end{aligned}
$$

Here we have used the notation

$$
|\nabla u(x, t)|^{2}=\sum_{i, j=1}^{n}\left|\frac{\partial u_{i}}{\partial x_{j}}\right|^{2} .
$$

It is easy to verify that the energy $E(t)$ decreases on $(0, \infty)$, but, in general, does not tend to zero as $t \rightarrow \infty$. In fact, it has been shown by Dafermos in his pioneering work [9] that the energy of every solution of (1.1) and (1.2) converges to zero as $t \rightarrow \infty$ if and only if $\Omega$ satisfies the following condition:

$\left(H_{\Omega}\right)$ There is no non-trivial eigenfunction $\phi=\phi(x)$ of the Lamé system

$$
-\mu \Delta \phi-(\lambda+\mu) \nabla \operatorname{div} \phi=\gamma^{2} \phi \quad \text { in } \Omega ; \quad \phi=0 \text { on } \partial \Omega
$$

such that $\operatorname{div} \phi=0$ in $\Omega$.

In [9], it was pointed out that $\left(H_{\Omega}\right)$ holds "generically" for smooth domains. It was also shown that $\left(H_{\Omega}\right)$ fails when $\Omega$ is a ball (see [32]).

Subsequently, the attention was paid to the problem of the uniform exponential decay rate of energy. In 1992, Hansen [12] made the first attempt on this problem by considering the one dimensional linear thermoelastic system subject to Dirichlet-Neumann or Neumann-Dirichlet boundary conditions. Applying the analysis of nonharmonic Fourier series, he succeeded in establishing the uniform exponential decay rate.

Hansen's results rely significantly on the boundary conditions of DirichletNeumann or Neumann-Dirichlet type. The case of Dirichlet-Dirichlet type was left as an open problem. Later, by using different methods, Kim [14] and Liu and Zheng [35] independently solved this problem, showing the exponential decay under Dirichlet-Dirichlet boundary conditions. While Kim used the energy method, multiplier techniques and compactness properties, Liu and Zheng's method was based on an abstract theorem about the exponential stability of semigroups.

Applying the abstract exponential stability theorem mentioned above, Burns, Liu and Zheng [5] further considered all other possible boundary conditions for the 1-D system of thermoelasticity (such as stress free at both ends and stress free at one end) and showed that the semigroups associated with these boundary conditions are also exponentially stable. 
In summary, the problem of exponential decay rate of energy for the onedimensional linear thermoelastic system has been completely solved by now.

For the higher-dimensional linear thermoelastic system, the problem is much more complicated. Since the total energy in higher-dimensional linear thermoelasticity does not always decay to zero, one has to try to partition the total energy into a dissipative part and a conservative part.

The question of partition of the energy was first studied by Lax and Phillips [23] for a classical solution of the wave equation. The first work about the partition of the energy in the linear thermoelasticity is due to Dassios and Grillakis [10], who studied how the energy associated with the longitudinal and thermal wave is divided into kinetic, strain, and thermal energy in the case $\Omega=\mathbb{R}^{3}$. They concluded that all three parts of the energy decay to zero as $t \rightarrow+\infty$ at a polynomial rate. Further, Rivera [43] studied the decomposition of the displacement vector field in $\mathbb{R}^{n}(n>1)$ into two parts. One of them is the solenoidal part, namely, the nondissipative component that conserves its energy and the other the dissipative component that decays to zero as fast as $t^{-n / 2}$ when $t$ approaches infinity.

For bounded domains, Chirita [8] proved that the mean thermal energy tends to zero as time goes to infinity and that the asymptotic equipartition occurs between the Cesàro means of the kinetic and strain energies. This shows that thermal effects do not influence explicitly the asymptotic equipartition of the mean kinetic and strain energies. Chirita's method relies on the Lagrange-Brun identities.

In special situations where the restoring force is proportional to the vector velocity of the displacement vector field, Pereira and Menzala [42] proved that in a bounded domain the kinetic, strain and thermal energies tend to zero exponentially as $t \rightarrow+\infty$.

Recently, Lebeau and Zuazua [25] gave a sufficient and necessary condition ensuring that the energy tends to zero exponentially as $t \rightarrow+\infty$ in a bounded multi-dimensional smooth domain $\Omega$. This condition is written in terms of the dynamics of the rays of geometric optics. As a consequence of the result of [25], it follows that when $\Omega$ is a bounded smooth convex open set, the energy does not decay exponentially to zero.

In addition, there has been a lot of work on von Kármán's system of thermoelastic plates. For details, we refer to [3], [6], [20], [22], [34], [36], [37], [38].

While there has been extensive work on the stabilization of the linear thermoelasticity, relatively little is known about the controllability.

The earliest results appear to be in the paper [40] of Narukawa, who proved the partial exact boundary controllability for the general form of the thermoelastic system on a bounded domain $\Omega$ in $\mathbb{R}^{n}$. Later, this result was improved by Lions [30, p. 32-60] by introducing the Hilbert Uniqueness Method. In both Narukawa and Lions' results, only the displacement is controlled, disregarding the values of the temperature. This is the so-called partial controllability property. Such a partial controllability property was also proved for von Kármán's system of thermoelastic plates (see [20], [21], [22]). This drawback (i.e., lack of information on the controllability of the temperature) was avoided by Hansen [13], who showed that, for at least 
the one-dimensional thermoelastic system, exact controllability of both the displacement and temperature is possible by only controlling the thermal or mechanical component on the boundary in the case where $u$ and $\theta$ satisfy the Dirichlet-Neumann or Neumann-Dirichlet boundary conditions and the coupling parameters satisfy some further restrictions. Hansen's results were proved by making use of the moment problems and the theory of nonharmonic Fourier series. It seems that Hansen's method is not applicable to the multi-dimensional space case. Thus, the problem of exact controllability of both the displacement and temperature is much more complicated in this case. In order to attack this problem, Zuazua [48] recently introduced the concept of exact-approximate controllability and made significant progress. He proved that, if $T$ is large enough, then the thermoelastic system is exact-approximately controllable with a control supported in a neighborhood of the boundary of $\Omega$, i.e., the displacement is shown to be exactly controllable and the temperature approximately controllable. The method of Zuazua combines multiplier techniques, compactness arguments and Holmgren's Uniqueness Theorem among other tools. More recently, Teresa and Zuazua [47] proved that the same kind of results hold for thermoelastic plates. In addition, Lebeau and Zuazua [26] proved that the system of linear thermoelasticity with periodic boundary conditions is null controllable with a volume force located in a subset satisfying the geometric control condition of [4]. Their method of proof is based on a spectral decomposition of the system and its adjoint on the basis generated by the eigenfunctions of the Laplacian. The spectrum is split into a parabolic and a hyperbolic part, and then the techniques of [4] and [24] are combined.

In this paper, we establish a sufficient condition which guarantees the exponential decay rate of the energy by means of an additional boundary damping. It is well known that the reason why the energy $E(t)$ does not tend to zero as $t \rightarrow \infty$ is that the total energy is not dissipated completely in the form of thermal energy (see [25] for more details). Thus we introduce here a velocity feedback on part of the boundary of the thermoelastic body, which is clamped along the rest of the boundary, to increase the loss of energy.

In order to state the boundary velocity feedback, we set

$$
\begin{aligned}
& \Gamma_{1}=\{x \in \Gamma: m(x) \cdot \nu(x) \leq 0\}, \\
& \Gamma_{2}=\{x \in \Gamma: m(x) \cdot \nu(x)>0\},
\end{aligned}
$$

where $m(x)=x-x^{0}=\left(x_{1}-x_{1}^{0}, \cdots, x_{n}-x_{n}^{0}\right)$ for some $x^{0} \in \mathbb{R}^{n}, \nu=$ $\left(\nu_{1}, \cdots, \nu_{n}\right)$ denotes the unit normal on $\Gamma$ directed towards the exterior of $\Omega$ and

$$
m \cdot \nu=m(x) \cdot \nu(x)=\sum_{i=1}^{n}\left(x_{i}-x_{i}^{0}\right) \nu_{i} .
$$

$\Gamma_{1}$ is assumed either to be empty or to have a nonempty interior relative to $\Gamma$. Note that assumptions (1.4) and (1.5) imply that the domain $\Omega$ is simply connected and star-shaped with respect to $x^{0} \in \Omega$ or $\Omega=\Omega_{1}-\bar{\Omega}_{2}$, both $\Omega_{1}$ and $\Omega_{2}$ being star-shaped with respect to $x^{0}$. Especially, the domain $\Omega$ 
can be a bounded smooth convex open set. As mentioned before, for the convex domain, Lebeau and Zuazua [25] has showed that the energy does not decay exponentially to zero in general. Thus, the feedback is necessary for this case.

The boundary velocity feedback can be stated as follows

$$
\begin{cases}\theta=0 & \text { on } \Gamma \times(0, \infty), \\ u=0 & \text { on } \Gamma_{1} \times(0, \infty), \\ \mu \frac{\partial u}{\partial \nu}+(\lambda+\mu) \operatorname{div}(u) \nu & \\ +a m \cdot \nu u+m \cdot \nu u^{\prime}=0 & \text { on } \Gamma_{2} \times(0, \infty),\end{cases}
$$

where $a=a(x)$ is a given nonegative function on $\Gamma_{2}$ with

$$
a(x) \in C^{1}\left(\Gamma_{2}\right) .
$$

It is clear that if $m(x) \cdot \nu(x) \geq \eta$ on $\Gamma_{2}$ for some $\eta>0$ then $a(x) m(x) \cdot \nu(x)$ can be any nonnegative function as we can take $a(x)=f(x) /(m(x) \cdot \nu(x))$, $f(x)$ being any nonnegative function. It is well known that the boundary velocity feedback is an effective mechanism to increase the loss of energy, and has been extensively used for the wave equation [7], [16], [18], elastodynamic systems [2], [19] and viscoelasticity [27], [28].

We will prove that the energy of the solutions of (1.1) and (1.6) decays to zero exponentially as $t \rightarrow \infty$ if $A=\max _{x \in \Gamma_{2}} a(x)$ is small enough (see Theorem 2.1 below).

In the special case of the Lamé system

$$
\begin{cases}u^{\prime \prime}-\mu \Delta u-(\lambda+\mu) \nabla \operatorname{div} u=0 & \text { in } \Omega \times(0, \infty), \\ u=0 & \text { on } \Gamma_{1} \times(0, \infty), \\ \mu \frac{\partial u}{\partial \nu}+(\lambda+\mu) \operatorname{div}(u) \nu & \\ \quad+a m \cdot \nu u+m \cdot \nu u^{\prime}=0 & \text { on } \Gamma_{2} \times(0, \infty), \\ u(0)=u^{0}, u^{\prime}(0)=u^{1} & \text { in } \Omega,\end{cases}
$$

the exponential stability still holds even though $A$ is large. This result answers an open question raised by Alabau and Komornik [2].

On the other hand, as the consequence of the uniform stabilization, we use the "Controllability via Stabilizability" principle to prove the partial exact boundary controllability for the thermoelastic system without smallness restrictions on the coupling parameters $\alpha$ and $\beta$.

The main results of this paper are presented in Section 2 and proved in Section 3. The methods of our proofs are based on multiplier techniques, the asymptotic property of the semigroups and Russell's "Controllability via Stabilizability" principle.

\section{MAin RESULTS}

In what follows, $H^{s}(\Omega)$ denotes the usual Sobolev space (see [1]) and $\|\cdot\|_{s}$ denotes its norm for any $s \in \mathbb{R}$. For $s \geq 0, H_{0}^{s}(\Omega)$ denotes the completion of 
$C_{0}^{\infty}(\Omega)$ in $H^{s}(\Omega)$, where $C_{0}^{\infty}(\Omega)$ denotes the space of all infinitely differentiable functions on $\Omega$ with compact supports in $\Omega$. Let $X$ be a Banach space. We denote by $C^{k}([0, T] ; X)$ the space of all $k$ times continuously differentiable functions defined on $[0, T]$ with values in $X$, and write $C([0, T] ; X)$ for $C^{0}([0, T] ; X)$.

Let $a(x)$ be the nonnegative function given in (1.6) satisfying (1.7) and $\nu=\left(\nu_{1}, \cdots, \nu_{n}\right)$ the unit normal on $\Gamma$ directed towards the exterior of $\Omega$. Suppose that $\Gamma_{1}$ and $\Gamma_{2}$ are given by (1.4) and (1.5), respectively, and $\Gamma_{1}$ either is empty or has a nonempty interior relative to $\Gamma$. Set

$$
\begin{gathered}
H_{\Gamma_{1}}^{1}(\Omega)=\left\{u \in H^{1}(\Omega): u=0 \text { on } \Gamma_{1}\right\} \\
H_{\Gamma_{1}}^{2}(\Omega)=\left\{u \in H^{2}(\Omega): u=0 \text { on } \Gamma_{1}\right\} \\
V=\left\{(u, v) \in\left(H^{1}(\Omega)\right)^{n} \times\left(L^{2}(\Omega)\right)^{n}: \int_{\Gamma} m \cdot \nu u d \Gamma+\int_{\Omega} v d x=0\right\}, \\
W= \begin{cases}V, & \text { if } \Gamma_{1}=\emptyset \text { and } a(x) \equiv 0, \\
\left(H_{\Gamma_{1}}^{1}(\Omega)\right)^{n} \times\left(L^{2}(\Omega)\right)^{n}, & \text { otherwise } \\
\mathcal{H}=W \times L^{2}(\Omega) .\end{cases}
\end{gathered}
$$

Further, if $\Gamma_{1}=\emptyset$ and $a(x) \equiv 0$, we set

$$
\begin{aligned}
D_{\Gamma_{1}}=\left\{(u, v, \theta): \theta \in\left(H^{2}(\Omega) \cap H_{0}^{1}(\Omega)\right),\right. & (u, v) \in\left(\left(H^{2}(\Omega)\right)^{n} \times\left(H^{1}(\Omega)\right)^{n} \cap V,\right. \\
& \left.\mu \frac{\partial u}{\partial \nu}+(\lambda+\mu) \operatorname{div}(u) \nu+a m \cdot \nu u+m \cdot \nu v=0 \text { on } \Gamma_{2}\right\} .
\end{aligned}
$$

Otherwise, we set

$$
\begin{aligned}
D_{\Gamma_{1}}=\left\{(u, v, \theta): \theta \in\left(H^{2}(\Omega) \cap H_{0}^{1}(\Omega)\right),\right. \\
(u, v) \in\left(H_{\Gamma_{1}}^{2}(\Omega)\right)^{n} \times\left(H_{\Gamma_{1}}^{1}(\Omega)\right)^{n}, \\
\left.\mu \frac{\partial u}{\partial \nu}+(\lambda+\mu) \operatorname{div}(u) \nu+a m \cdot \nu u+m \cdot \nu v=0 \text { on } \Gamma_{2}\right\} .
\end{aligned}
$$

Note that the norm on $W$

$$
\begin{aligned}
\|(u, v)\|_{W}= & \left(\frac{1}{2} \int_{\Omega}\left[\mu|\nabla u|^{2}+(\lambda+\mu)|\operatorname{div}(u)|^{2}+|v|^{2}\right] d x\right. \\
& \left.+\frac{1}{2} \int_{\Gamma_{2}} a m \cdot \nu|u|^{2} d \Gamma\right)^{1 / 2}
\end{aligned}
$$

is equivalent to the usual one induced by $\left(H^{1}(\Omega)\right)^{n} \times\left(L^{2}(\Omega)\right)^{n}$. When proving this, the only delicate case is when $\Gamma_{1}=\emptyset$ and $a(x) \equiv 0$. We argue by contradiction. If the norms are not equivalent, then there is a sequence $\left\{\left(u^{n}, v^{n}\right)\right\} \in V$ such that

$$
\int_{\Omega}\left[\left|\nabla u^{n}\right|^{2}+\left|u^{n}\right|^{2}+\left|v^{n}\right|^{2}\right] d x=1,
$$


and

$$
\int_{\Omega}\left|u^{n}\right|^{2} d x \geq n \int_{\Omega}\left[\mu\left|\nabla u^{n}\right|^{2}+(\lambda+\mu)\left|\operatorname{div}\left(u^{n}\right)\right|^{2}+\left|v^{n}\right|^{2}\right] d x .
$$

Then, we have

$$
\begin{aligned}
& \lim _{n \rightarrow \infty} \int_{\Omega}\left|\nabla u^{n}\right|^{2} d x=0, \\
& \lim _{n \rightarrow \infty} \int_{\Omega}\left|v^{n}\right|^{2} d x=0 .
\end{aligned}
$$

Moreover, by (2.2), we may assume that $\left\{u^{n}\right\}$ converges to $u$ weakly in $\left(H^{1}(\Omega)\right)^{n}$. Since the injection of $\left(H^{1}(\Omega)\right)^{n}$ into $\left(L^{2}(\Omega)\right)^{n}$ is compact, we may assume that $\left\{u^{n}\right\}$ converges to $u$ strongly in $\left(L^{2}(\Omega)\right)^{n}$. Since in the sense of distribution

$$
\lim _{n \rightarrow \infty} \frac{\partial u^{n}}{\partial x_{i}}=\frac{\partial u}{\partial x_{i}}
$$

(2.4) implies that

$$
\frac{\partial u}{\partial x_{i}}=0 .
$$

Thus

$$
u=C \text { (constant). }
$$

Because $\left(u^{n}, v^{n}\right) \in V$, we have

$$
\int_{\Gamma} m \cdot \nu u^{n} d \Gamma+\int_{\Omega} v^{n} d x=0 .
$$

By (2.5), we deduce that

$$
\lim _{n \rightarrow \infty} \int_{\Omega} v^{n} d x=0
$$

It therefore follows from (2.6) that

$$
\lim _{n \rightarrow \infty} \int_{\Gamma} m \cdot \nu u^{n} d \Gamma=0 .
$$

On the other hand, by (2.4) and the fact that $\left\{u^{n}\right\}$ converges to $C$ strongly in $\left(L^{2}(\Omega)\right)^{n}$, we can deduce that $\left\{u^{n}\right\}$ converges to $C$ strongly in $\left(H^{1}(\Omega)\right)^{n}$. Thus, by the trace theorem, we have

$$
C \int_{\Gamma} m \cdot \nu d \Gamma=\lim _{n \rightarrow \infty} \int_{\Gamma} m \cdot \nu u^{n} d \Gamma=0 .
$$

This shows that $C=0$. Consequently, it follows from (2.4) and (2.5) that

$$
\lim _{n \rightarrow \infty} \int_{\Omega}\left[\left|\nabla u^{n}\right|^{2}+\left|u^{n}\right|^{2}+\left|v^{n}\right|^{2}\right] d x=0
$$

which is in contradiction to $(2.2)$. 
If $a(x) \not \equiv 0$, the proof is similar. If $\Gamma_{1} \neq \emptyset$, this is just the consequence of Poincaré inequality (see [1, p. 159]).

In the sequel, we use the energy norm on $\mathcal{H}$ :

$$
\begin{aligned}
\|(u, v, \theta)\|_{\mathcal{H}}= & \left(\frac{1}{2} \int_{\Omega}\left[\mu|\nabla u|^{2}+(\lambda+\mu)|\operatorname{div}(u)|^{2}+|v|^{2}+\frac{\alpha}{\beta}|\theta|^{2}\right] d x\right. \\
& \left.+\frac{1}{2} \int_{\Gamma_{2}} a m \cdot \nu|u|^{2} d \Gamma\right)^{1 / 2}
\end{aligned}
$$

for $(u, v, \theta) \in \mathcal{H}$, which is equivalent to the usual one induced by $\left(H^{1}(\Omega)\right)^{n} \times$ $\left(L^{2}(\Omega)\right)^{n} \times L^{2}(\Omega)$.

We consider the thermoelastic system with a velocity feedback:

$$
\begin{cases}u^{\prime \prime}-\mu \Delta u-(\lambda+\mu) \nabla \operatorname{div} u+\alpha \nabla \theta=0 & \text { in } \Omega \times(0, \infty), \\ \theta^{\prime}-\Delta \theta+\beta \operatorname{div} u^{\prime}=0 & \text { in } \Omega \times(0, \infty), \\ \theta=0 & \text { on } \Gamma \times(0, \infty) \\ u=0 & \text { on } \Gamma_{1} \times(0, \infty), \\ \mu \frac{\partial u}{\partial \nu}+(\lambda+\mu) \operatorname{div}(u) \nu & \\ \quad+a m \cdot \nu u+m \cdot \nu u^{\prime}=0 & \text { on } \Gamma_{2} \times(0, \infty), \\ u(0)=u^{0}, u^{\prime}(0)=u^{1}, \theta(0)=\theta^{0} & \text { in } \Omega .\end{cases}
$$

It is well known that problem (2.8) is well-posed (see [40]). In fact, the system generates a strongly continuous semigroup $S(t)$ in $\mathcal{H}$. This has been proved in [33] for the more general case of thermoviscoelasticity. However, the case $\Gamma_{1}=\emptyset$ and $a(x) \equiv 0$ is worth discussing in more detail. Indeed, from previous articles published in the literature, one may think that system (2.8) generates a semigroup in the following space with zero average:

$$
\begin{array}{r}
\mathcal{H}_{0}=\left\{(u, v, \theta) \in\left(H^{1}(\Omega)\right)^{n} \times\left(L^{2}(\Omega)\right)^{n} \times L^{2}(\Omega):\right. \\
\left.\int_{\Omega} u(x) d x=\int_{\Omega} v(x) d x=0\right\} .
\end{array}
$$

Actually this is a mistake. To see this, we define the function $f(t)$ by

$$
f(t)=\int_{\Omega} u(t) d x
$$

Take $\left(u^{0}, u^{1}, \theta^{0}\right) \in \mathcal{H}_{0}$ such that

$$
\int_{\Gamma} m \cdot \nu u^{0} d \Gamma \neq 0
$$

and let $u, \theta$ be the solution of (2.8) corresponding to this initial data. We claim that

$$
\int_{\Gamma} m \cdot \nu u^{\prime}(t) d \Gamma \not \equiv 0 .
$$

If this is not true, then $\int_{\Gamma} m \cdot \nu u(t) d \Gamma$ is constant in time. In addition, we can show that the energy $\left\|\left(u(t), u^{\prime}(t), \theta(t)\right)\right\|_{\mathcal{H}}^{2}$ decays to zero exponentially 
as $t \rightarrow \infty$. Consequently, $\int_{\Gamma} m \cdot \nu u(t) d \Gamma$ also decays to zero exponentially since there is a positive constant $C$ such that

$$
\left|\int_{\Gamma} m \cdot \nu u(t) d \Gamma\right| \leq C\left\|\left(u(t), u^{\prime}(t), \theta(t)\right)\right\|_{\mathcal{H}} .
$$

It therefore follows that

$$
\int_{\Gamma} m \cdot \nu u(t) d \Gamma \equiv 0,
$$

which contradicts (2.9). By (2.10), we have

$$
\begin{aligned}
f^{\prime \prime}(t) & =\int_{\Omega} u^{\prime \prime}(t) d x \\
& =\int_{\Omega}[\mu \Delta u+(\lambda+\mu) \nabla \operatorname{div} u-\alpha \nabla \theta] d x \\
& =\int_{\Gamma}\left[\mu \frac{\partial u}{\partial \nu}+(\lambda+\mu) \operatorname{div}(u) \nu-\alpha \theta \nu\right] d \Gamma \\
& =-\int_{\Gamma} m \cdot \nu u^{\prime}(t) d \Gamma \\
& \neq 0 .
\end{aligned}
$$

Hence, $\int_{\Omega} u(t) d x$ and $\int_{\Omega} u^{\prime}(t) d x$ are not always equal to zero along the solution trajectories of $(2.8)$. Thus, $\mathcal{H}_{0}$ is not invariant under the flow given by (2.8). Consequently, system (2.8) does not generate a semigroup in $\mathcal{H}_{0}$.

On the other hand, $\mathcal{H}$ is invariant under the flow given by (2.8). In fact, the function

$$
g(t)=\int_{\Gamma} m \cdot \nu u(t) d \Gamma+\int_{\Omega} u^{\prime}(t) d x
$$

is constant along the solution trajectories of (2.8). This is because

$$
\begin{aligned}
g^{\prime}(t) & =\int_{\Gamma} m \cdot \nu u^{\prime}(t) d \Gamma+\int_{\Omega} u^{\prime \prime}(t) d x \\
& =\int_{\Gamma} m \cdot \nu u^{\prime}(t) d \Gamma+\int_{\Omega}[\mu \Delta u+(\lambda+\mu) \nabla \operatorname{div} u-\alpha \nabla \theta] d x \\
& =\int_{\Gamma} m \cdot \nu u^{\prime}(t) d \Gamma+\int_{\Gamma}\left[\mu \frac{\partial u}{\partial \nu}+(\lambda+\mu) \operatorname{div}(u) \nu-\alpha \theta \nu\right] d \Gamma \\
& =0
\end{aligned}
$$

This is why the chosen space for solving $(2.8)$ is $\mathcal{H}$.

Generally speaking, one can say that a semigroup preserves a quantity if and only if its generator does it. More precisely, let us consider

$$
\left\{\begin{array}{l}
u^{\prime}=A u, \quad t>0 \\
u(0)=u_{0},
\end{array}\right.
$$

on a Banach space $X$, where $A$ is a unbounded linear operator on $X$ with the domain $D(A)$ and $u_{0} \in X$. Assume that $A$ generates a semigroup. let 
$L: X \rightarrow \mathbb{R}$ be a linear and bounded functional. Then, the following two conditions are equivalent:

(i) $L u(t)$ remains constant in time for every solution $u$ of (2.11);

(ii) $L\left(A u_{0}\right)=0$ for all $u_{0} \in D(A)$.

To see that (ii) implies (i), we first assume that $u_{0} \in D(A)$, and then $u(t) \in D(A)$ for every $t \geq 0$. Applying $L$ to equation (2.11), we obtain

$$
\frac{d L(u(t))}{d t}=L\left(u^{\prime}\right)=L(A(u(t))=0 .
$$

Therefore, $L(u(t))$ remains constant in time. If $u_{0} \in X$, by density, we can show that (i) still holds.

For the other implication, note that $A u_{0}$ is the limit of $(u(t)-u(0)) / t$ in $X$ as $t \rightarrow 0, u$ being the solution of the equation with initial data $u_{0}$. Now, in view of (i), we have $L((u(t)-u(0)) / t)=0$ for all $t$. On the other hand, this quantity should converge to $L\left(A u_{0}\right)$ as $t \rightarrow 0$. This shows that (ii) holds.

Note that, in our situation, the functional $L$ is given by

$$
L(u, v)=\int_{\Gamma} m \cdot \nu u d \Gamma+\int_{\Omega} v d x
$$

Since system (2.8) generates a strongly continuous semigroup $S(t)$ in $\mathcal{H}$, then for every initial data

$$
\left(u^{0}, u^{1}, \theta^{0}\right) \in \mathcal{H}
$$

system $(2.8)$ has a unique solution $\left(u, u^{\prime}, \theta\right)$ with

$$
\left(u, u^{\prime}, \theta\right) \in C([0, \infty) ; \mathcal{H}) .
$$

Moreover, if

$$
\left(u^{0}, u^{1}, \theta^{0}\right) \in D_{\Gamma_{1}},
$$

then

$$
\left(u, u^{\prime}, \theta\right) \in C\left([0, \infty) ; D_{\Gamma_{1}}\right) .
$$

In order to ensure that the solution $u$ has sufficient regularity to perform the integrations by parts we will do, in this paper, we suppose that

$$
\bar{\Gamma}_{1} \cap \bar{\Gamma}_{2}=\emptyset
$$

Under this assumption, by the standard elliptic regularity properties, we have

$$
u \in C\left([0, \infty) ;\left(H^{2}(\Omega)\right)^{n}\right) .
$$

This regularity property is needed for the proof of the following theorems. If (2.12) does not hold, then (2.13) fails in general even in the case of the wave equation (see [18]). However, even though (2.12) fails, Komornik and Zuazua [16] still proved the uniform boundary stabilization of the wave equation in the case where $n \leq 3$. Their proof was based on an inequality established 
by Grisvard [11] for the solution of the wave equation with such boundary singularity. Whether or not Komornik and Zuazua's result still holds for the system of thermoelasticity with such boundary singularity is an open problem as the similar inequality of Grisvard has not been proved yet in the literature.

In order to state our main results, we introduce some constants as follows. Set

$$
\begin{gathered}
R\left(x^{0}\right)=\max _{x \in \bar{\Omega}}|m(x)|=\max _{x \in \bar{\Omega}}\left|\sum_{k=1}^{n}\left(x_{k}-x_{k}^{0}\right)^{2}\right|^{1 / 2}, \\
K(a)=\frac{2 A^{2} R\left(x^{0}\right)^{2}}{\mu}+(2-n) A,
\end{gathered}
$$

where $m(x)=x-x^{0}$ and $A=\max _{x \in \Gamma_{2}} a(x)$. Let $\gamma$ be the smallest positive constant such that

$$
\int_{\Gamma_{2}}|u|^{2} d \Gamma \leq \gamma^{2}\|u\|_{H^{1}(\Omega)}^{2}, \quad \forall u \in H^{1}(\Omega) .
$$

Let $\lambda_{0}$ be the smallest positive constant such that

$$
\|(u, v)\|_{H^{1}(\Omega) \times L^{2}(\Omega)} \leq \lambda_{0}\|(u, v)\|_{W}, \quad \forall(u, v) \in W .
$$

The thermoelastic energy of (2.8) is defined by

$$
E(u, \theta, t)=\left\|\left(u(t), u^{\prime}(t), \theta(t)\right)\right\|_{\mathcal{H}}^{2} .
$$

We now state our main results of this paper.

Theorem 2.1. Let $\Gamma_{1}$ and $\Gamma_{2}$ be given by (1.4) and (1.5), respectively, satisfying (2.12). If the function a $(x)$ satisfies

$$
K(a) R\left(x^{0}\right) \gamma^{2} \lambda_{0}^{2}<2, \quad \text { for } n \leq 2,
$$

or

$$
a(x) \leq \frac{(n-2) \mu}{2 R^{2}\left(x^{0}\right)} \quad \text { on } \quad \Gamma_{2}, \quad \text { for } n \geq 3,
$$

then there exists a positive constant $\omega$, independent of $\left(u^{0}, u^{1}, \theta^{0}\right)$, such that

$$
E(u, \theta, t) \leq E(u, \theta, 0) e^{1-\omega t}, \quad \forall t \geq 0,
$$

for all solutions of (2.8) with $\left(u^{0}, u^{1}, \theta^{0}\right) \in \mathcal{H}$. Further, the constant $\omega$ can be given by

$$
\omega=k^{-1}
$$

where

$$
\begin{gathered}
k=\left\{\begin{array}{cc}
\frac{k_{1}+k_{2}+k_{3}}{2-K(a) R\left(x^{0}\right) \gamma^{2} \lambda_{0}^{2}-\varepsilon\left(R\left(x^{0}\right) \gamma^{2} \lambda_{0}^{2}+\frac{2}{\mu}+\lambda_{0}^{2}\right)}, & n \leq 2, \\
\frac{k_{1}+k_{2}+k_{3}}{2-\varepsilon\left(R\left(x^{0}\right) \gamma^{2} \lambda_{0}^{2}+\frac{2}{\mu}+\lambda_{0}^{2}\right)}, & n \geq 3,
\end{array}\right. \\
k_{1}=\frac{2 R^{2}\left(x^{0}\right)}{\mu}+\frac{(n-1)^{2}}{4 \varepsilon}+1,
\end{gathered}
$$




$$
\begin{gathered}
k_{2}=4 \max \left\{2, \frac{2 R^{2}\left(x^{0}\right)}{\mu}, \frac{(n-1)^{2} \lambda_{0}^{2}}{4}\right\}, \\
k_{3}=\frac{\alpha \beta R^{2}\left(x^{0}\right)}{\varepsilon}+\frac{\alpha \beta(n-1)^{2}}{4 \varepsilon}+\lambda_{0}^{2}, \\
0<\varepsilon< \begin{cases}\frac{2-K(a) R\left(x^{0}\right) \gamma^{2} \lambda_{0}^{2}}{R\left(x^{0}\right) \gamma^{2} \lambda_{0}^{2}+\frac{2}{\mu}+\lambda_{0}^{2}}, & n \leq 2, \\
\frac{2}{R\left(x^{0}\right) \gamma^{2} \lambda_{0}^{2}+\frac{2}{\mu}+\lambda_{0}^{2}}, & n \geq 3 .\end{cases}
\end{gathered}
$$

In the case of the Lamé system (1.8), Theorem 2.1 still holds even though $a$ is large. Namely, we have

Theorem 2.2. Let $\Gamma_{1}$ and $\Gamma_{2}$ be given by (1.4) and (1.5), respectively, satisfying (2.12). Let $a(x)$ be any nonnegative function on $\Gamma_{2}$ satisfying (1.7). Then there are positive constants $M, \omega$, independent of $\left(u^{0}, u^{1}\right)$, such that

$$
E(u, t) \leq M E(u, 0) e^{-\omega t}, \quad \forall t \geq 0,
$$

for all solutions of (1.8) with $\left(u^{0}, u^{1}\right) \in W$.

In this case, the energy $E(u, t)$ is given by

$$
\begin{aligned}
E(u, t)= & \frac{1}{2} \int_{\Omega}\left[\left|u^{\prime}(x, t)\right|^{2}+\mu|\nabla u(x, t)|^{2}+(\lambda+\mu)|\operatorname{div} u(x, t)|^{2}\right] d x \\
& +\frac{1}{2} \int_{\Gamma_{2}} a m \cdot \nu|u(t)|^{2} d \Gamma
\end{aligned}
$$

If the function $a(x)$ satisfies (2.19) or (2.20), then the constants $M$ and $\omega$ in Theorem 2.2, of course, are the same as in Theorem 2.1. However, if $a(x)$ does not satisfy (2.19) and (2.20), then $M$ and $\omega$ can not be explicitly given since in this case the proof of Theorem 2.2 is based on a control-theoretic method (see [39] and [46]), which is not constructive.

Theorem 2.2 is an answer to the open question raised by Alabau and Komornik [2], who proved the exponential stability of the Lamé system for the case where $a(x) m \cdot \nu \equiv a_{0}$ is a small enough constant and $\Omega$ is a ball, and then conjectured that this result probably remains true even for $a_{0}$ large.

We now consider the controllability problem:

$$
\begin{cases}u^{\prime \prime}-\mu \Delta u-(\lambda+\mu) \nabla \operatorname{div} u+\alpha \nabla \theta=0 & \text { in } \Omega \times(0, \infty), \\ \theta^{\prime}-\Delta \theta+\beta \operatorname{div} u^{\prime}=0 & \text { in } \Omega \times(0, \infty), \\ \theta=0 & \text { on } \Gamma \times(0, \infty), \\ u=0 & \text { on } \Gamma_{1} \times(0, \infty), \\ \mu \frac{\partial u}{\partial \nu}+(\lambda+\mu) \operatorname{div}(u) \nu+a m \cdot \nu u=\phi & \text { on } \Gamma_{2} \times(0, \infty), \\ u(0)=0, u^{\prime}(0)=0, \quad \theta(0)=0 & \text { in } \Omega,\end{cases}
$$

where $\phi=\left(\phi_{1}, \cdots, \phi_{n}\right)$ is a control acting on the boundary $\Gamma_{2}$.

Given $T>0$, let $Q=\Omega \times(0, T), \Sigma=\Gamma \times(0, T), \Sigma_{1}=\Gamma_{1} \times(0, T)$ and $\Sigma_{2}=\Gamma_{2} \times(0, T)$.

The partial exact controllability problem can be stated as follows: Given $T>0$, for every state $\left(u^{0}, u^{1}\right) \in W$, we want to find a control $\phi$ in a suitable function space such that the solution of (2.30) satisfies

$$
u(x, T)=u^{0}(x), u^{\prime}(x, T)=u^{1}(x) \quad \text { in } \Omega,
$$


disregarding the values of the temperature.

As stated in [30, p. 34-35], this is equivalent to steering every initial state $\left(u^{0}, u^{1}\right)$ of the displacement in the function space to the state $\left(u(T), u^{\prime}(T)\right)=$ $(0,0)$, disregarding the values of the temperature.

Let $\sigma$ be the smallest positive constant such that

$$
\|\operatorname{div} u\|_{-1} \leq \sigma\|u\|_{0}, \quad \forall u \in L^{2}(\Omega) .
$$

Theorem 2.3. Let $\Gamma_{1}$ and $\Gamma_{2}$ be given by (1.4) and (1.5), respectively, satisfying (2.12). Assume that the function a (x) satisfies (2.19) or (2.20). Let $T_{0}$ be large enough such that if $T \geq T_{0}$ then

$$
2\left(1+\alpha^{2} \beta^{2} \sigma^{2} T^{2}\right) e^{1-\omega T}<1,
$$

where $\omega$ is the constant in Theorem 2.1. Then for any $\left(u^{0}, u^{1}\right) \in W$, there exists a boundary control function $\phi(x, t)$ with

$$
\frac{\phi}{\sqrt{m \cdot \nu}} \in\left(L^{2}\left(\Sigma_{2}\right)\right)^{n}
$$

such that the solution of (2.30) satisfies (2.31). Moreover, there exists positive constant $c$, independent of $\left(u^{0}, u^{1}\right)$, such that

$$
\left\|\frac{\phi}{\sqrt{m \cdot \nu}}\right\|\left(L^{2}\left(\Sigma_{2}\right)\right)^{n} \leq c\left\|\left(u^{0}, u^{1}\right)\right\|_{W} .
$$

In comparison with the existing literature, the main contribution of Theorem 2.3 is that there is no smallness restriction on the coupling parameters $\alpha$ and $\beta$.

In the case of the Lamé system

$$
\begin{cases}u^{\prime \prime}-\mu \Delta u-(\lambda+\mu) \nabla \operatorname{div} u=0 & \text { in } \Omega \times(0, \infty) \\ u=0 & \text { on } \Gamma_{1} \times(0, \infty), \\ \mu \frac{\partial u}{\partial \nu}+(\lambda+\mu) \operatorname{div}(u) \nu+a m \cdot \nu u=\phi & \text { on } \Gamma_{2} \times(0, \infty), \\ u(0)=0, u^{\prime}(0)=0 & \text { in } \Omega,\end{cases}
$$

Theorem 2.3 still holds even though $a$ is large. Namely, we have

Theorem 2.4. Let $\Gamma_{1}$ and $\Gamma_{2}$ be given by (1.4) and (1.5), respectively, satisfying (2.12). Let $a(x)$ be any nonnegative function on $\Gamma_{2}$ satisfying (1.7). Let $T_{0}$ be large enough such that if $T \geq T_{0}$ then

$$
M e^{-\omega T}<1 \text {, }
$$

where $M$ and $\omega$ are the constants in Theorem 2.2. Then for any $\left(u^{0}, u^{1}\right) \in$ $W$, there exists a boundary control function $\phi(x, t)$ with

$$
\frac{\phi}{\sqrt{m \cdot \nu}} \in\left(L^{2}\left(\Sigma_{2}\right)\right)^{n}
$$

such that the solution of (2.35) satisfies (2.31). Moreover, there exist positive constants $c_{1}, c_{2}$, independent of $\left(u^{0}, u^{1}\right)$, such that

$$
c_{1}\left\|\left(u^{0}, u^{1}\right)\right\|_{W} \leq\left\|\frac{\phi}{\sqrt{m \cdot \nu}}\right\|_{\left(L^{2}\left(\Sigma_{2}\right)\right)^{n}} \leq c_{2}\left\|\left(u^{0}, u^{1}\right)\right\|_{W} .
$$




\section{Proof of main Results}

Let us first describe our steps of proof of Theorems 2.1-2.4. We first prove Theorem 2.1, and then prove Theorem 2.3. Since the Lamé system is a special case of the thermoelastic system, Theorems 2.2 and 2.4 are simultaneously proved in the case that $a(x)$ satisfies $(2.19)$ or (2.20). Finally, we prove Theorems 2.2 and 2.4 in the case that $a(x)$ is large (i.e., $a(x)$ does not necessarily satisfy $(2.19)$ or $(2.20))$.

In the sequel, the summation convention is assumed. We recall the constants $R\left(x^{0}\right), K(a), \gamma$ and $\lambda_{0}$ are given by (2.14)-(2.17), respectively.

Proof of Theorem 2.1. The idea of the proof of Theorem 2.1 is simple. According to Theorem 8.1 of $[15$, p. 103], it suffices to show that

$$
\int_{s}^{\infty} E(u, \theta, t) d t \leq k E(u, \theta, s), \quad \forall s \geq 0
$$

where $k$ is given by (2.23). However, the proof of this inequality is generally not easy. We use the multiplier techniques to attack it.

Given $0 \leq s<T$, let $Q_{s}=\Omega \times(s, T), \Sigma_{s}=\Gamma \times(s, T), \Sigma_{1 s}=\Gamma_{1} \times(s, T)$ and $\Sigma_{2 s}=\Gamma_{2} \times(s, T)$.

We may as well assume that $\left(u^{0}, u^{1}, \theta^{0}\right) \in D_{\Gamma_{1}}$ since the general case $\left(u^{0}, u^{1}, \theta^{0}\right) \in \mathcal{H}$ can be handled by density. Then $(u, \theta)$ is a classical solution of (2.8). Multiplying the first equation of (2.8) by $m_{k} \frac{\partial u_{i}}{\partial x_{k}}$ and integrating on $Q_{s}$, we have

$$
\begin{aligned}
\int_{Q_{s}} m_{k} \frac{\partial u_{i}}{\partial x_{k}} & u_{i}^{\prime \prime} d x d t=\left.\left(u_{i}^{\prime}(t), m_{k} \frac{\partial u_{i}(t)}{\partial x_{k}}\right)\right|_{s} ^{T}-\frac{1}{2} \int_{\Sigma_{s}} m_{k} \nu_{k}\left|u_{i}^{\prime}\right|^{2} d \Sigma \\
& +\frac{n}{2} \int_{Q_{s}}\left|u_{i}^{\prime}\right|^{2} d x d t \\
& \int_{Q_{s}} m_{k} \frac{\partial u_{i}}{\partial x_{k}} \Delta u_{i} d x d t \\
= & \int_{\Sigma_{s}} \frac{\partial u_{i}}{\partial \nu} m_{k} \frac{\partial u_{i}}{\partial x_{k}} d \Sigma-\int_{Q_{s}} \frac{\partial u_{i}}{\partial x_{j}} \frac{\partial}{\partial x_{j}}\left(m_{k} \frac{\partial u_{i}}{\partial x_{k}}\right) d x d t \\
= & \int_{\Sigma_{s}} \frac{\partial u_{i}}{\partial \nu} m_{k} \frac{\partial u_{i}}{\partial x_{k}} d \Sigma-\int_{Q_{s}}\left|\nabla u_{i}\right|^{2} d x d t \\
& -\frac{1}{2} \int_{Q_{s}} m_{k} \frac{\partial}{\partial x_{k}}\left|\nabla u_{i}\right|^{2} d x d t \\
= & \int_{\Sigma_{s}} \frac{\partial u_{i}}{\partial \nu} m_{k} \frac{\partial u_{i}}{\partial x_{k}} d \Sigma-\frac{1}{2} \int_{\Sigma_{s}} m_{k} \nu_{k}\left|\nabla u_{i}\right|^{2} d \Sigma \\
& +\frac{n-2}{2} \int_{Q_{s}}\left|\nabla u_{i}\right|^{2}
\end{aligned}
$$




$$
\begin{aligned}
& \int_{Q_{s}} m_{k} \frac{\partial u_{i}}{\partial x_{k}} \frac{\partial}{\partial x_{i}}(\operatorname{div} u) d x d t \\
& =\int_{\Sigma_{s}} \operatorname{div}(u) m_{k} \frac{\partial u_{i}}{\partial x_{k}} \nu_{i} d \Sigma-\int_{Q_{s}} \operatorname{div}(u) \frac{\partial}{\partial x_{i}}\left(m_{k} \frac{\partial u_{i}}{\partial x_{k}}\right) d x d t \\
& =\int_{\Sigma_{s}} \operatorname{div}(u) m_{k} \frac{\partial u_{i}}{\partial x_{k}} \nu_{i} d \Sigma-\int_{Q_{s}}|\operatorname{div}(u)|^{2} d x d t \\
& \quad-\frac{1}{2} \int_{Q_{s}} m_{k} \frac{\partial}{\partial x_{k}}\left(|\operatorname{div}(u)|^{2}\right) d x d t \\
& =\int_{\Sigma_{s}} \operatorname{div}(u) m_{k} \frac{\partial u_{i}}{\partial x_{k}} \nu_{i} d \Sigma-\frac{1}{2} \int_{\Sigma_{s}} m_{k} \nu_{k}|\operatorname{div}(u)|^{2} d \Sigma \\
& \quad+\frac{n-2}{2} \int_{Q_{s}}|\operatorname{div}(u)|^{2} d x d t .
\end{aligned}
$$

Multiplying the first equation of (2.8) by $u_{i}$ and integrating over $Q_{s}$, we obtain

$$
\begin{aligned}
& \int_{Q_{s}}\left(\left|u_{i}{ }^{\prime}\right|^{2}-\mu\left|\nabla u_{i}\right|^{2}-(\lambda+\mu)|\operatorname{div} u|^{2}\right) d x d t \\
& =\left.\left(u_{i}^{\prime}(t), u_{i}\right)\right|_{s} ^{T}+\alpha \int_{Q_{s}} u_{i} \frac{\partial \theta}{\partial x_{i}} d x d t \\
& \quad-\mu \int_{\Sigma_{s}} \frac{\partial u_{i}}{\partial \nu} u_{i} d \Sigma-(\lambda+\mu) \int_{\Sigma_{s}} u_{i} \nu_{i} \operatorname{div}(u) d \Sigma .
\end{aligned}
$$

It therefore follows from (2.8) and (3.1)-(3.4) that

$$
\begin{aligned}
& 2 \int_{s}^{T} E(u, \theta, t) d t \\
& =\int_{\Sigma_{s}} m_{k} \nu_{k}\left[\left|u_{i}^{\prime}\right|^{2}-\mu\left|\nabla u_{i}\right|^{2}-(\lambda+\mu)|\operatorname{div}(u)|^{2}\right] d \Sigma \\
& \quad+2 \int_{\Sigma_{s}}\left[\mu \frac{\partial u_{i}}{\partial \nu}+(\lambda+\mu) \operatorname{div}(u) \nu_{i}\right] m_{k} \frac{\partial u_{i}}{\partial x_{k}} d \Sigma \\
& \quad+(n-1) \int_{\Sigma_{s}}\left[\mu \frac{\partial u_{i}}{\partial \nu}+(\lambda+\mu) \operatorname{div}(u) \nu_{i}\right] u_{i} d \Sigma+\int_{\Sigma_{2 s}} a m \cdot \nu\left|u_{i}\right|^{2} d \Sigma \\
& \quad-\left.2\left(u_{i}^{\prime}(t), m_{k} \frac{\partial u_{i}(t)}{\partial x_{k}}+\frac{n-1}{2} u_{i}\right)\right|_{s} ^{T} \\
& \quad-2 \alpha \int_{Q_{s}} \nabla \theta\left(m_{k} \frac{\partial u}{\partial x_{k}}+\frac{n-1}{2} u\right) d x d t+\frac{\alpha}{\beta} \int_{Q_{s}}|\theta|^{2} d x d t
\end{aligned}
$$




$$
\begin{aligned}
& \text { (use } \frac{\partial u_{i}}{\partial x_{k}}=\frac{\partial u_{i}}{\partial \nu} \nu_{k} \text { on } \Sigma_{1 \mathrm{~s}} \text { and boundary conditions of (2.8)) } \\
& =\int_{\Sigma_{1 s}} m \cdot \nu\left[\mu\left|\frac{\partial u_{i}}{\partial \nu}\right|^{2}+(\lambda+\mu)|\operatorname{div}(u)|^{2}\right] d \Sigma\left(=I_{1}\right) \\
& \quad+\int_{\Sigma_{2 s}} m \cdot \nu\left[\left|u_{i}^{\prime}\right|^{2}-\mu\left|\nabla u_{i}\right|^{2}-(\lambda+\mu)|\operatorname{div}(u)|^{2}\right] d \Sigma\left(=I_{2}\right) \\
& \quad-2 \int_{\Sigma_{2 s}} m \cdot \nu\left[a u_{i}+u_{i}^{\prime}\right] m_{k} \frac{\partial u_{i}}{\partial x_{k}} d \Sigma\left(=I_{3}\right) \\
& \quad-(n-1) \int_{\Sigma_{2 s}} m \cdot \nu\left[a u_{i}+u_{i}^{\prime}\right] u_{i} d \Sigma+\int_{\Sigma_{2 s}} a m \cdot \nu\left|u_{i}\right|^{2} d \Sigma\left(=I_{4}\right) \\
& \quad-\left.2\left(u_{i}^{\prime}(t), m_{k} \frac{\partial u_{i}(t)}{\partial x_{k}}+\frac{n-1}{2} u_{i}\right)\right|_{s} ^{T}\left(=I_{5}\right) \\
& \quad-2 \alpha \int_{Q_{s}} \nabla \theta\left(m_{k} \frac{\partial u}{\partial x_{k}}+\frac{n-1}{2} u\right) d x d t+\frac{\alpha}{\beta} \int_{Q_{s}}|\theta|^{2} d x d t\left(=I_{6}\right) .
\end{aligned}
$$

We now estimate $I_{i}(i=1,2, \cdots, 6)$. Since $m \cdot \nu \leq 0$ on $\Gamma_{1}$, we have

$$
I_{1} \leq 0 \text {. }
$$

Using the inequality

$$
p q \leq \varepsilon p^{2}+\frac{1}{4 \varepsilon} q^{2}, \quad \forall p, q \geq 0, \varepsilon>0
$$

we have

$$
\begin{aligned}
I_{3} \leq & \frac{2 R^{2}\left(x^{0}\right)}{\mu} \int_{\Sigma_{2 s}} a^{2} m \cdot \nu\left|u_{i}\right|^{2} d \Sigma+\frac{2 R^{2}\left(x^{0}\right)}{\mu} \int_{\Sigma_{2 s}} m \cdot \nu\left|u_{i}^{\prime}\right|^{2} d \Sigma \\
& +\mu \int_{\Sigma_{2 s}} m \cdot \nu\left|\nabla u_{i}\right|^{2} d \Sigma,
\end{aligned}
$$

and

$$
\begin{aligned}
I_{4}= & -(n-1) \int_{\Sigma_{2 s}} m \cdot \nu u_{i}^{\prime} u_{i} d \Sigma+(2-n) \int_{\Sigma_{2 s}} a m \cdot \nu\left|u_{i}\right|^{2} d \Sigma \\
\leq & \frac{(n-1)^{2}}{4 \varepsilon} \int_{\Sigma_{2 s}} m \cdot \nu\left|u_{i}^{\prime}\right|^{2} d \Sigma+\varepsilon \int_{\Sigma_{2 s}} m \cdot \nu\left|u_{i}\right|^{2} d \Sigma \\
& +(2-n) \int_{\Sigma_{2 s}} a m \cdot \nu\left|u_{i}\right|^{2} d \Sigma \\
(\text { use }(2.16) \text { and }(2.17)) & \\
\leq & \frac{(n-1)^{2}}{4 \varepsilon} \int_{\Sigma_{2 s}} m \cdot \nu\left|u_{i}^{\prime}\right|^{2} d \Sigma+\varepsilon \gamma^{2} \lambda_{0}^{2} R\left(x^{0}\right) \int_{s}^{T} E(u, \theta, t) d t \\
& +(2-n) \int_{\Sigma_{2 s}} a m \cdot \nu\left|u_{i}\right|^{2} d \Sigma .
\end{aligned}
$$

In order to estimate $I_{5}$ and $I_{6}$, multiplying the first equation and second equation of (2.8) by $u_{i}^{\prime}$ and $\frac{\alpha}{\beta} \theta$, respectively, and integrating over $Q_{s}$, we obtain

$$
E(u, \theta, T)+\frac{\alpha}{\beta} \int_{Q_{s}}|\nabla \theta|^{2} d x d t+\int_{\Sigma_{2 s}} m \cdot \nu\left|u_{i}^{\prime}\right|^{2} d \Sigma=E(u, \theta, s)
$$


Noting definitions (2.25) and (2.26) of $k_{2}$ and $k_{3}$, it follows from (2.17) and (3.10) that

$$
I_{5} \leq k_{2} E(u, \theta, s)
$$

and

$$
\begin{aligned}
I_{6} \leq & \frac{\alpha^{2} R^{2}\left(x^{0}\right)}{\varepsilon} \int_{Q_{s}}|\nabla \theta|^{2} d x d t+\varepsilon \int_{Q_{s}}\left|\nabla u_{i}\right|^{2} d x d t \\
& +\frac{\alpha^{2}(n-1)^{2}}{4 \varepsilon} \int_{Q_{s}}|\nabla \theta|^{2} d x d t+\varepsilon \int_{Q_{s}}\left|u_{i}\right|^{2} d x d t \\
& +\frac{\alpha \lambda_{0}^{2}}{\beta} \int_{Q_{s}}|\nabla \theta|^{2} d x d t \\
\leq & k_{3} E(u, \theta, s)+\varepsilon\left(\frac{2}{\mu}+\lambda_{0}^{2}\right) \int_{s}^{T} E(u, \theta, t) d t .
\end{aligned}
$$

Noting definition (2.24) of $k_{1}$, it then follows from (3.5)-(3.12) that

$$
\begin{aligned}
& 2 \int_{s}^{T} E(u, \theta, t) d t \\
& \leq k_{1} \int_{\Sigma_{2 s}} m \cdot \nu\left|u_{i}^{\prime}\right|^{2}+\left(k_{2}+k_{3}\right) E(u, \theta, s) \\
& +\varepsilon\left(\gamma^{2} \lambda_{0}^{2} R\left(x^{0}\right)+\frac{2}{\mu}+\lambda_{0}^{2}\right) \int_{s}^{T} E(u, \theta, t) d t \\
& +\int_{\Sigma_{2 s}}\left[\frac{2 R^{2}\left(x^{0}\right) a^{2}}{\mu}+(2-n) a\right] m \cdot \nu\left|u_{i}\right|^{2} d \Sigma \quad(=I) \\
& \left.\leq\left(k_{1}+k_{2}+k_{3}\right) E(u, \theta, s) \quad \text { (use }(3.10)\right) \\
& +\varepsilon\left(\gamma^{2} \lambda_{0}^{2} R\left(x^{0}\right)+\frac{2}{\mu}+\lambda_{0}^{2}\right) \int_{s}^{T} E(u, \theta, t) d t \\
& +I \text {. }
\end{aligned}
$$

If $n \geq 3$, then, by (2.20), we deduce that $I \leq 0$. Thus, if $\varepsilon$ satisfies $(2.27)$, then we deduce from (3.13) that

$$
\int_{s}^{T} E(u, \theta, t) d t \leq k E(u, \theta, s), \quad \forall 0 \leq s<T,
$$

where $k$ is given by (2.23).

If $n \leq 2$, then we have

$$
I \leq K(a) R\left(x^{0}\right) \gamma^{2} \lambda_{0}^{2} \int_{s}^{T} E(u, \theta, t) d t .
$$

It therefore follows from (2.19) and (3.13) that (3.14) also holds provided $\varepsilon$ satisfies (2.27). Then, by Theorem 8.1 of [15, p. 103], we deduce that

$$
E(u, \theta, t) \leq E(u, \theta, 0) e^{(k-t) / k}, \quad \forall t \geq 0 .
$$

This completes the proof of Theorem 2.1. 
Note that Theorem 2.2 is simultaneously proved in the case that $a(x)$ satisfies (2.19) or (2.20) since the Lamé system is a special case of the thermoelastic system. This fact will be used in the following proof of Theorem 2.3 .

We now use Russell's "Controllability via Stabilizability" principle [45] to prove Theorem 2.3.

Proof of Theorem 2.3. Given $\left(v^{0}, v^{1}\right) \in W$, we consider the Lamé system

$$
\begin{cases}v^{\prime \prime}-\mu \Delta v-(\lambda+\mu) \nabla \operatorname{div} v=0 & \text { in } Q, \\ v=0 & \text { on } \Sigma_{1} \\ \mu \frac{\partial v}{\partial \nu}+(\lambda+\mu) \operatorname{div}(v) \nu+a m \cdot \nu v-m \cdot \nu v^{\prime}=0 & \text { on } \Sigma_{2} \\ v(T)=v^{0}, \quad v^{\prime}(T)=v^{1} & \text { in } \Omega\end{cases}
$$

which has a unique solution with

$$
\left(v(t), v^{\prime}(t)\right) \in C([0, T] ; W) .
$$

Moreover, by Theorem 2.2 (note that we are assuming that $a(x)$ satisfies (2.19) or (2.20), and in this case, Theorem 2.2 has been proved as the special case of the thermoelastic system), there exists a positive constant $\omega$ such that

$$
E(v, t) \leq E(v, T) e^{1-\omega(T-t)}, \quad \forall t \in[0, T]
$$

where

$$
\begin{aligned}
E(v, t)= & \frac{1}{2} \int_{\Omega}\left[\left|v^{\prime}(x, t)\right|^{2}+\mu|\nabla v(x, t)|^{2}+(\lambda+\mu)|\operatorname{div} v(x, t)|^{2}\right] d x \\
& +\frac{1}{2} \int_{\Gamma_{2}} a m \cdot \nu|v(t)|^{2} d \Gamma
\end{aligned}
$$

Using the solution $v$ of $(3.15)$, we then consider

$$
\begin{cases}\xi^{\prime}-\Delta \xi=\beta \operatorname{div}\left(v^{\prime}\right) & \text { in } Q \\ \xi=0 & \text { on } \Sigma \\ \xi(0)=0 & \text { in } \Omega\end{cases}
$$

and

$$
\begin{cases}w^{\prime \prime}-\mu \Delta w-(\lambda+\mu) \nabla \operatorname{div} w+\alpha \nabla \psi=-\alpha \nabla \xi & \text { in } Q \\ \psi^{\prime}-\Delta \psi+\beta \operatorname{div} w^{\prime}=0 & \text { in } Q \\ \psi=0 & \text { on } \Sigma \\ w=0 & \text { on } \Sigma_{1} \\ \mu \frac{\partial w}{\partial \nu}+(\lambda+\mu) \operatorname{div}(w) \nu+a m \cdot \nu w+m \cdot \nu w^{\prime}=0 & \text { on } \Sigma_{2} \\ w(0)=v(0), \quad w^{\prime}(0)=v^{\prime}(0), \quad \psi(0)=0 & \text { in } \Omega\end{cases}
$$

Since

$$
\operatorname{div}\left(v^{\prime}\right) \in\left(L^{2}\left(0, T ; H^{-1}(\Omega)\right)\right)^{n},
$$

it follows that (3.17) has a unique solution $\xi$ with

$$
\xi \in C\left([0, T] ; L^{2}(\Omega)\right) \cap L^{2}\left(0, T ; H_{0}^{1}(\Omega)\right) .
$$


In addition, multiplying (3.17) by $\xi$ and integrating over $Q$, we obtain

$$
\begin{aligned}
& \frac{1}{2}\|\xi(T)\|_{0}^{2}+\int_{0}^{T}\|\nabla \xi(t)\|_{0}^{2} d t \\
& =\beta \int_{0}^{T} \int_{\Omega} \operatorname{div}\left(v^{\prime}\right) \xi d x d t \\
& \leq \beta \int_{0}^{T}\left\|\operatorname{div}\left(v^{\prime}(t)\right)\right\|_{-1}\|\nabla \xi(t)\|_{0} d t \quad(\text { use }(2.32)) \\
& \left.\leq \frac{1}{2} \int_{0}^{T}\|\nabla \xi(t)\|_{0}^{2} d t+\frac{\beta^{2} \sigma^{2}}{2} \int_{0}^{T}\left\|v^{\prime}(t)\right\|_{0}^{2} d t \quad \text { (use }(3.16)\right) \\
& \leq \frac{1}{2} \int_{0}^{T}\|\nabla \xi(t)\|_{0}^{2} d t+\frac{\beta^{2} \sigma^{2}}{2} T e^{1-\omega T} E(v, T)
\end{aligned}
$$

which implies

$$
\|\xi(T)\|_{0}^{2}+\int_{0}^{T}\|\nabla \xi(t)\|_{0}^{2} d t \leq \beta^{2} \sigma^{2} T e^{1-\omega T} E(v, T) .
$$

On the other hand, since $\xi \in L^{2}\left(0, T ; H_{0}^{1}(\Omega)\right)$ and

$$
\int_{\Omega} \nabla \xi d x=\int_{\Gamma} \nu \xi d \Gamma=0
$$

then $(0,-\alpha \nabla \xi, 0) \in L^{1}(0, T ; \mathcal{H})$. Thus, by the classical theory of semigroups, the nonhomogeneous problem (3.18) has a unique solution with

$$
\left(w, w^{\prime}, \psi\right) \in C([0, T] ; \mathcal{H}) .
$$

Moreover, the solution can be expressed as

$$
\left(w, w^{\prime}, \psi\right)=S(t)\left(w(0), w^{\prime}(0), \psi(0)\right)+\int_{0}^{t} S(t-\tau)(0,-\alpha \nabla \xi, 0) d \tau
$$

where $S(t)$ denotes the strongly continuous semigroup of contractions generated by the thermoelastic system. Since the semigroup $S(t)$ is contractive, that is,

$$
\|S(t)\| \leq 1, \quad \forall t \geq 0
$$

then we deduce from (3.19)

$$
\begin{aligned}
E(w, \psi, t) & \leq 2 E(w, \psi, 0)+2\left(\int_{0}^{t}\|\alpha \nabla \xi(\tau)\|_{0} d \tau\right)^{2} \\
& \leq 2 E(w, \psi, 0)+2 \alpha^{2} \beta^{2} \sigma^{2} T^{2} e^{1-\omega T} E(v, T)
\end{aligned}
$$

Set

$$
u=w-v, \quad \theta=\psi+\xi
$$

and

$$
\phi=-m \cdot \nu\left(w^{\prime}+v^{\prime}\right)
$$


then $u, \theta$ satisfies

$$
\begin{cases}u^{\prime \prime}-\mu \Delta u-(\lambda+\mu) \nabla \operatorname{div} u+\alpha \nabla \theta=0 & \text { in } Q, \\ \theta^{\prime}-\Delta \theta+\beta \operatorname{div} u^{\prime}=0 & \text { in } Q, \\ \theta=0 & \text { on } \Sigma, \\ u=0 & \text { on } \Sigma_{1}, \\ \mu \frac{\partial u}{\partial \nu}+(\lambda+\mu) \operatorname{div}(u) \nu+a m \cdot \nu u=\phi & \text { on } \Sigma_{2}, \\ u(0)=u^{\prime}(0)=0, \quad \theta(0)=0 & \text { in } \Omega, \\ u(T)=w(T)-v^{0}, \quad u^{\prime}(T)=w^{\prime}(T)-v^{1} & \text { in } \Omega .\end{cases}
$$

We define an operator $\Lambda$ by

$$
\Lambda\left(v^{0}, v^{1}\right)=\left(w(T), w^{\prime}(T)\right)
$$

Then it is clear that $\Lambda$ is a linear operator from $W$ into $W$. Moreover, by (3.16) and (3.20), we have

$$
\begin{aligned}
& \left\|\Lambda\left(v^{0}, v^{1}\right)\right\|_{W}^{2} \\
& \text { (note definition }(2.1) \text { of the norm of } W) \\
& \leq E(w, \psi, T) \\
& \leq 2 E(w, \psi, 0)+2 \alpha^{2} \beta^{2} \sigma^{2} T^{2} e^{1-\omega T} E(v, T) \\
& \text { (since } \left.w(0)=v(0), w^{\prime}(0)=v^{\prime}(0), \psi(0)=0\right) \\
& =2 E(v, 0)+2 \alpha^{2} \beta^{2} \sigma^{2} T^{2} e^{1-\omega T} E(v, T) \\
& \leq\left(2+2 \alpha^{2} \beta^{2} \sigma^{2} T^{2}\right) e^{1-\omega T} E(v, T) \quad(\text { use }(3.16)) \\
& =\left(2+2 \alpha^{2} \beta^{2} \sigma^{2} T^{2}\right) e^{1-\omega T}\left\|\left(v^{0}, v^{1}\right)\right\|_{W}^{2}
\end{aligned}
$$

Therefore,

$$
\|\Lambda\|^{2} \leq\left(2+2 \alpha^{2} \beta^{2} \sigma^{2} T^{2}\right) e^{1-\omega T} .
$$

Let $T_{0}$ be large enough so that (2.33) holds if $T \geq T_{0}$. Then $\Lambda-I$ is an isomorphism from $W$ onto $W$. Thus, for any $\left(u^{0}, u^{1}\right) \in W$, there exists a unique $\left(v^{0}, v^{1}\right) \in W$ such that

$$
\begin{aligned}
\left(u^{0}, u^{1}\right) & =\Lambda\left(v^{0}, v^{1}\right)-\left(v^{0}, v^{1}\right) \\
& =\left(w(T), w^{\prime}(T)\right)-\left(v^{0}, v^{1}\right) \\
& =\left(u(T), u^{\prime}(T)\right) .
\end{aligned}
$$

Consequently, we have constructed a control function $\phi=-m \cdot \nu\left(w^{\prime}+v^{\prime}\right)$ solving the partial controllability problem (2.30).

On the other hand, multiplying the first equation of (3.15) by $v_{i}^{\prime}$ and integrating over $Q$, we obtain

$$
\int_{\Sigma_{2}} m \cdot \nu\left|v^{\prime}\right|^{2} d \Sigma=E(v, T)-E(v, 0) .
$$


Multiplying the first equation and second equation of (3.18) by $w_{i}^{\prime}$ and $\frac{\alpha}{\beta} \psi$, respectively, and integrating over $Q$, we deduce from (3.19) and (3.20) that (the following $c$ 's denoting various constants)

$$
\begin{aligned}
& E(w, \psi, T)+\frac{\alpha}{\beta} \int_{Q}|\nabla \psi|^{2} d x d t+\int_{\Sigma_{2}} m \cdot \nu\left|w^{\prime}\right|^{2} d \Sigma \\
& =E(w, \psi, 0)-\alpha \int_{Q} w^{\prime} \cdot \nabla \xi d x d t \\
& \leq E(w, \psi, 0)+c T e^{-\omega T} E(v, T)+\int_{0}^{T} E(w, \psi, \tau) d \tau \\
& \leq(1+2 T) E(w, \psi, 0)+\left(1+T^{2}\right) c T e^{-\omega T} E(v, T) .
\end{aligned}
$$

Noting $E(v, 0)=E(w, \psi, 0)$, we deduce from (3.16), (3.23) and (3.24) that

$$
\int_{\Sigma_{2}} \frac{|\phi|^{2}}{m \cdot \nu} d \Sigma=\int_{\Sigma_{2}} m \cdot \nu\left|v^{\prime}+w^{\prime}\right|^{2} d \Sigma \leq c E(v, T),
$$

which, combining with (3.22), implies (2.34) since $\Lambda-I$ is an isomorphism from $W$ onto itself.

Note that Theorem 2.4 is simultaneously proved in the case that $a(x)$ satisfies (2.19) or (2.20) since the Lamé system is a special case of the thermoelastic system. This fact will be used in the following proof of Theorem 2.2 .

We now use the control-theoretic method given in [39] and [46] to complete the proof of Theorem 2.2. That is, we are going to prove Theorem 2.2 in the case that $a(x)$ is large.

Proof of Theorem 2.2. Let $u(t)$ be the solution of (1.8). Let $\varepsilon>0$ satisfy (2.19) or (2.20) and let $T$ be large enough so that Theorem 2.4 holds with $a=\varepsilon$ (note that we are assuming that $a$ satisfies (2.19) or (2.20), and in this case, Theorem 2.4 has been proved as the special case of the thermoelastic system). Since $\left(u(T), u^{\prime}(T)\right) \in W$, it follows from Theorem 2.4 that there exists a control $\phi$ such that

$$
\begin{cases}y^{\prime \prime}-\mu \Delta y-(\lambda+\mu) \nabla \operatorname{div} y=0 & \text { in } \Omega \times(0, \infty), \\ y=0 & \text { on } \Gamma_{1} \times(0, \infty), \\ \mu \frac{\partial y}{\partial \nu}+(\lambda+\mu) \operatorname{div}(y) \nu+\varepsilon m \cdot \nu y=\phi & \text { on } \Gamma_{2} \times(0, \infty), \\ y(0)=0, \quad y^{\prime}(0)=0 & \text { in } \Omega, \\ y(T)=u(T), y^{\prime}(T)=u^{\prime}(T) & \text { in } \Omega .\end{cases}
$$

According to the proof of Theorem 2.3, $y$ and $\phi$ can be written as

$$
y=w-v, \quad \phi=-m \cdot \nu\left(v^{\prime}+w^{\prime}\right),
$$

where $v$ and $w$ are respectively the solutions of

$$
\begin{cases}v^{\prime \prime}-\mu \Delta v-(\lambda+\mu) \nabla \operatorname{div} v=0 & \text { in } Q \\ v=0 & \text { on } \Sigma_{1} \\ \mu \frac{\partial v}{\partial \nu}+(\lambda+\mu) \operatorname{div}(v) \nu+\varepsilon m \cdot \nu v-m \cdot \nu v^{\prime}=0 & \text { on } \Sigma_{2} \\ v(T)=v^{0}, \quad v^{\prime}(T)=v^{1} & \text { in } \Omega\end{cases}
$$


and

$$
\begin{cases}w^{\prime \prime}-\mu \Delta w-(\lambda+\mu) \nabla \operatorname{div} w=0 & \text { in } \Omega \times(0, \infty), \\ w=0 & \text { on } \Gamma_{1} \times(0, \infty), \\ \mu \frac{\partial w}{\partial \nu}+(\lambda+\mu) \operatorname{div}(w) \nu+\varepsilon m \cdot \nu w+m \cdot \nu w^{\prime}=0 & \text { on } \Gamma_{2} \times(0, \infty), \\ w(0)=v(0), \quad w^{\prime}(0)=v^{\prime}(0) & \text { in } \Omega .\end{cases}
$$

Note that the Lamé system is the special case of the thermoelastic system where $\alpha=\beta=0$, so (3.18) is reduced to (3.28). In $(3.27),\left(v^{0}, v^{1}\right)$ is chosen to be such that

$$
\left(w(T), w^{\prime}(T)\right)-\left(v^{0}, v^{1}\right)=\Lambda\left(v^{0}, v^{1}\right)-\left(v^{0}, v^{1}\right)=\left(u(T), u^{\prime}(T)\right) .
$$

To avoid confusion, we denote the energy of (1.8) with $a(x)$ by $E_{a}(u, t)$ and the energy of $(3.27)$ with $\varepsilon$ by $E_{\varepsilon}(v, t)$. Evidently, $E_{a}(u, t)$ is equivalent to $E_{\varepsilon}(u, t)$.

Integrating by parts, it follows from (1.8) and (3.25) that

$$
\begin{aligned}
0= & \int_{Q}\left[y_{i}^{\prime}\left(u_{i}^{\prime \prime}-\mu \Delta u_{i}-(\lambda+\mu) \nabla \operatorname{div} u\right)\right. \\
& \left.+u_{i}^{\prime}\left(y_{i}^{\prime \prime}-\mu \Delta y_{i}-(\lambda+\mu) \nabla \operatorname{div} y\right)\right] d x d t \\
= & \int_{Q} \frac{\partial}{\partial t}\left(u_{i}^{\prime} y_{i}^{\prime}+\mu \nabla u_{i} \cdot \nabla y_{i}+(\lambda+\mu) \operatorname{div} u \operatorname{div} y\right) d x d t \\
& +\int_{\Sigma_{2}}\left(y_{i}^{\prime} m \cdot \nu\left(a u_{i}+u_{i}^{\prime}\right)+u_{i}^{\prime}\left(\varepsilon m \cdot \nu y_{i}-\phi_{i}\right)\right) d \Sigma \\
= & \int_{\Omega}\left(\left|u_{i}^{\prime}(T)\right|^{2}+\mu\left|\nabla u_{i}(T)\right|^{2}+(\lambda+\mu)|\operatorname{div} u(T)|^{2}\right) d x \\
& +\int_{\Gamma_{2}} a m \cdot \nu\left|u_{i}(T)\right|^{2} d \Gamma \\
& +\int_{\Sigma_{2}} u_{i}^{\prime}\left(m \cdot \nu y_{i}^{\prime}-a m \cdot \nu y_{i}+\varepsilon m \cdot \nu y_{i}-\phi_{i}\right) d \Sigma \\
= & 2 E_{a}(u, T)+\int_{\Sigma_{2}} u_{i}^{\prime}\left(m \cdot \nu y_{i}^{\prime}-a m \cdot \nu y_{i}+\varepsilon m \cdot \nu y_{i}-\phi_{i}\right) d \Sigma .
\end{aligned}
$$

By the trace theorem and (3.26) and (3.29), we have (the following $c$ 's denoting various constants that may depend on $T$ )

$$
\begin{aligned}
\int_{\Sigma_{2}} m \cdot \nu|y|^{2} d \Sigma & \leq c \int_{0}^{T}\|y(t)\|_{\left(H^{1}(\Omega)\right)^{n}}^{2} d t \\
& \leq c \int_{0}^{T}\left(\|v\|_{\left(H^{1}(\Omega)\right)^{n}}^{2}+\|w\|_{\left(H^{1}(\Omega)\right)^{n}}^{2}\right) d t \\
& \leq c\left(E_{\varepsilon}(v, T)+E_{\varepsilon}(w, 0)\right) \\
& \leq c E_{\varepsilon}(v, T) \quad\left(E_{\varepsilon}(w, 0)=E_{\varepsilon}(v, 0) \leq c E_{\varepsilon}(v, T)\right) \\
& \leq c E_{\varepsilon}(u, T) \quad(\text { use }(3.29)) \\
& \leq c E_{a}(u, T) .
\end{aligned}
$$


Multiplying the first equation of (3.27) by $v_{i}^{\prime}$ and integrating over $Q$, we obtain

$$
\int_{\Sigma_{2}} m \cdot \nu\left|v^{\prime}\right|^{2} d \Sigma=E_{\varepsilon}(v, T)-E_{\varepsilon}(v, 0)
$$

Similarly, we have

$$
\int_{\Sigma_{2}} m \cdot \nu\left|w^{\prime}\right|^{2} d \Sigma=E_{\varepsilon}(w, 0)-E_{\varepsilon}(w, T) .
$$

It therefore follows from (3.26) and (3.29) that

$$
\begin{aligned}
& \int_{\Sigma_{2}} m \cdot \nu\left|y^{\prime}\right|^{2} d \Sigma \\
& \leq 2 \int_{\Sigma_{2}} m \cdot \nu\left(\left|v^{\prime}\right|^{2}+\left|w^{\prime}\right|^{2}\right) d \Sigma \\
& \leq c\left[E_{\varepsilon}(v, T)-E_{\varepsilon}(v, 0)+E_{\varepsilon}(w, 0)-E_{\varepsilon}(w, T)\right] \\
& \leq c E_{\varepsilon}(v, T) \quad(\text { as in }(3.31)) \\
& \leq c E_{a}(u, T) .
\end{aligned}
$$

By (2.37) (note that we are assuming that $a=\varepsilon$ satisfies (2.19) or (2.20), and in this case, Theorem 2.4 has been proved as the special case of the thermoelastic system, so (2.37) can be used), we have

$$
\int_{\Sigma_{2}} \frac{|\phi|^{2}}{m \cdot \nu} d \Sigma \leq c E_{\varepsilon}(u, T) \leq c E_{a}(u, T) .
$$

By Cauchy-Schwarz's inequality we deduce from (3.30) that

$$
\left(E_{a}(u, T)\right)^{2} \leq c \int_{\Sigma_{2}} m \cdot \nu\left|u_{i}^{\prime}\right|^{2} d \Sigma \int_{\Sigma_{2}} m \cdot \nu\left|y_{i}^{\prime}-a y_{i}+\varepsilon y_{i}-\frac{\phi_{i}}{m \cdot \nu}\right|^{2} d \Sigma
$$

It therefore follows from $(3.31),(3.34)$ and $(3.35)$ that

$$
c E_{a}(u, T) \leq \int_{\Sigma_{2}} m \cdot \nu\left|u^{\prime}\right|^{2} d \Sigma .
$$

On the other hand, multiplying the first equation of (1.8) by $u_{i}^{\prime}$ and integrating over $Q$, we obtain

$$
\int_{\Sigma_{2}} m \cdot \nu\left|u^{\prime}\right|^{2} d \Sigma=E_{a}(u, 0)-E_{a}(u, T)
$$

We then conclude from (3.37) and (3.38) that

$$
c E_{a}(u, T) \leq E_{a}(u, 0)-E_{a}(u, T),
$$

and hence,

$$
E_{a}(u, T) \leq \frac{1}{1+c} E_{a}(u, 0)
$$


Repeating the above reasoning, we get

$$
\begin{aligned}
E_{a}(u,(k+1) T) & \leq \frac{1}{1+c} E_{a}(u, k T) \\
& \leq \frac{1}{(1+c)^{k+1}} E_{a}(u, 0), \quad k=0,1,2, \cdots
\end{aligned}
$$

which implies (2.28) with

$$
M=1+c, \quad \omega=\frac{1}{T} \ln (1+c) .
$$

Proof of Theorem 2.4. The proof is the same as that of Theorem 2.3 except that (3.18) is replaced by

$$
\begin{cases}w^{\prime \prime}-\mu \Delta w-(\lambda+\mu) \nabla \operatorname{div} w=0 & \text { in } Q \\ w=0 & \text { on } \Sigma_{1} \\ \mu \frac{\partial w}{\partial \nu}+(\lambda+\mu) \operatorname{div}(w) \nu+a m \cdot \nu w+m \cdot \nu w^{\prime}=0 & \text { on } \Sigma_{2} \\ w(0)=v(0), \quad w^{\prime}(0)=v^{\prime}(0) & \text { in } \Omega .\end{cases}
$$

In this case, we obtain

$$
\|\Lambda\| \leq M e^{-\omega T}
$$

Thus, (2.33) becomes (2.36). In addition, for Theorem 2.4, we have to further prove the following estimate

$$
c_{1}\left\|\left(u^{0}, u^{1}\right)\right\|_{W} \leq\left\|\frac{\phi}{\sqrt{m \cdot \nu}}\right\|_{\left(L^{2}\left(\Sigma_{2}\right)\right)^{n}} .
$$

Multiplying the first equation of (3.39) by $w_{i}^{\prime}$ and integrating over $Q$, we obtain

$$
\int_{\Sigma_{2}} m \cdot \nu\left|w^{\prime}\right|^{2} d \Sigma=E(w, 0)-E(w, T) .
$$

It therefore follows from Theorem 2.2 that

$$
\left(1-M e^{-\omega T}\right)^{1 / 2} E^{1 / 2}(w, 0) \leq\left\|\sqrt{m \cdot \nu} w^{\prime}\right\|_{L^{2}\left(\Sigma_{2}\right)} \leq E^{1 / 2}(w, 0) .
$$

Similarly, by (4.23), we deduce that

$$
\left(1-M e^{-\omega T}\right)^{1 / 2} E^{1 / 2}(v, T) \leq\left\|\sqrt{m \cdot \nu} v^{\prime}\right\|_{L^{2}\left(\Sigma_{2}\right)} \leq E^{1 / 2}(v, T) .
$$

Noting $E(v, 0)=E(w, 0)$, we deduce from the triangle inequality and Theorem 2.2 that

$$
\begin{aligned}
& {\left[1-M e^{-\omega T}\right]^{1 / 2}\left[1-\left(M e^{-\omega T}\right)^{1 / 2}\right] E^{1 / 2}(v, T)} \\
& \leq\left\|\sqrt{m \cdot \nu}\left(v^{\prime}+w^{\prime}\right)\right\|_{L^{2}\left(\Sigma_{2}\right)} \\
& \leq\left[1+\left(M e^{-\omega T}\right)^{1 / 2}\right] E^{1 / 2}(v, T) .
\end{aligned}
$$

Since $I-\Lambda$ is an isomorphism, (3.40) follows from (3.21), (3.22) and (3.44). 
I am deeply indebted to Professor E. Zuazua for his relevant advice. Also I thank my supervisor Dr. Graham Williams for his encouragement in the preparation of this paper. Finally, I am grateful for the financial support from the Overseas Postgraduate Research Scholarship from Australian Government, the University of Wollongong Postgraduate Research Award, and the Department of Mathematics Analysis Research Group Scholarship from the Faculty of Informatics at the University of Wollongong.

\section{REFERENCES}

[1] R. Adams: Sobolev spaces, Academic Press, New York, 1975.

[2] F. Alabau, V. Komornik: Boundary observability, controllability and stabilization of linear elastodynamic systems, SIAM J. Control Optim., to appear.

[3] G. Avalos, I. Lasiecka: Exponential stability of a free thermoelastic system without mechanical dissipation, SIAM J. Math. Anal., to appear.

[4] C. Bardos, G. Lebeau, J. Rauch: Sharp sufficient conditions for the observation, control, and stabilization of waves from the boundary, SIAM J. Control Optim. 30, 1992, 1024-1065.

[5] J.A. Burns, Z.Y. Liu, S. Zheng: On the energy decay of a linear thermoelastic bar, J. Math. Anal. Appl., 179, 1993, 574-591.

[6] E. Bisognin, V. Bisognin, G.P. Menzala, E. Zuazua: On exponential stability for the Von Kármán equations in the presence of thermal effects, Math. Methods Appl. Sci, to appear.

[7] G. Chen: Energy decay estimates and exact boundary value controllability for the wave equation in a bounded domain, J. Math. Pures Appl., 58, 1979, 249-273.

[8] S. Chirita: On the asymptotic partition of the energy in linear thermoelasticity, Quart. Appl. Math., 45, 1987, 327-340.

[9] C.M. Dafermos: On the existence and the asymptotic stability of solution to the equations of linear thermoelasticity, Arch. Rational Mech. Anal., 29, 1968, 241-271.

[10] G. Dassios, M. Grillakis: Dissipation rates and partition of energy in thermoelasticity, Arch. Rational Mech. Anal., 87, 1984, 49-91.

[11] P. Grisvard: Contrôlabilité exacte des solutions de l'équation des ondes en présence de singularités, J. Math. Pures Appl., 68, 1989, 215-259.

[12] S.W. Hansen: Exponential energy decay in a linear thermoelastic rod, J. Math. Anal. Appl., 167, 1992, 429-442.

[13] S.W. Hansen: Boundary control of a one-dimensional linear thermoelastic rod, SIAM J. Control Optim., 32, 1994, 1054-1074.

[14] J.U. Kim: On the energy decay of a linear thermoelastic bar and plate, SIAM J. Math. Anal., 23, 1992, 889-899.

[15] V. Komornik: Exact controllability and stabilization: The multiplier method, John Wiley \& Sons, Masson, Paris, 1994.

[16] V. Komornik, E. Zuazua: A direct method for the boundary stabilization of the wave equation, J. Math. Pures Appl., 69, 1990, 33-54.

[17] A.D. Kovalenko: Thermoelasticity, basic theory and applications, Wolters-Noordhoff Publishing, Groningen, Netherlands, 1969.

[18] J. Lagnese: Decay of solutions of wave equations in a bounded region with boundary dissipation, J. Differential Equations, 50, 1983, 163-182.

[19] J. Lagnese: Boundary stabilization of linear elastodynamic systems, SIAM J. Control Optim., 21, 1983, 968-984.

[20] J. Lagnese: Boundary Stabilization of Thin Plates, in SIAM Studies in Applied Mathematics, 10, SIAM Publications, Philadelphia, 1989.

[21] J. Lagnese: The reachability problem for thermoelastic plates, Arch. Rational Mech. Anal., 112 (1990), 223-267.

[22] J. Lagnese, J-.L. Lions: Modelling analysis and control of thin plates, Masson, Paris, 1989. 
[23] P. Lax, R.S. Phillips: Scattering theory, Revised Edition, Academic Press INC., Boston, 1989.

[24] G. Lebeau, L. Robbiano: Contrôle exact de l'équation de la chaleur, Comm. Partial Differential Equations, 20 (1 \& 2), 1995, 335-356.

[25] G. Lebeau, E. Zuazua: Sur la décroissance non uniforme de l'énergie dans le système de la thermoélasticité linéaire, C. R. Acad. Sci. Paris Sér. I Math., 324, 1997, 409415 .

[26] G. Lebeau, E. Zuazua: Null controllability of a system of linear thermoelasticity, Arch. Rational Mech. Anal., to appear.

[27] G. Leugering: On boundary feedback stabilization of a viscoelastic membrance, $D y$ nam. Stability Systems, 4, 1989, 71-79.

[28] G. Leugering: On boundary feedback stabilization of a viscoelastic beam, Proc. Roy. Soc. Edinburgh Sect. A, 114, 1990, 57-69.

[29] G. Leugering: A decomposition method for integro-partial differential equations and applications, J. Math. Pures Appl., 71, 1992, 561-587.

[30] J.-L. Lions: Contrôlabilité exacte perturbations et stabilisation de systèmes distribués, Tome 2, Perturbations, Masson, Paris, 1988.

[31] J.-L. Lions, E. Magenes: Non-homogeneous boundary value problems and applications, vol. I and II, Springer-Verlag, New York, 1972.

[32] J.-L. Lions, E. Zuazua, A generic Uniqueness Result for the Stokes system and its control theoretical consequences, in Partial Differential Equations and Applications, P. Marcellini, G.T. Talenti, E. Vesentini, Eds., Lecture Notes in Pure and Applied Mathematics, 177, 221-235, Marcel Dekker, Inc., New York, 1996.

[33] W.J. Liu, The exponential stabilization of the higher-dimensional linear thermoviscoelasticity, J. Math. Pures Appl., to appear.

[34] Z. Liu, M. Renardy: A note on the equations of thermoelastic plates, Appl. Math. Letters, 8, 1995, 1-6.

[35] Z. Liu, S. Zheng: Exponential stability of the semigroup associated with a thermoelastic system, Quart. Appl. Math., LI, 1993, 535-545.

[36] Z. Liu, S. Zheng: Exponential stability of the Kirchoff plate with thermal or viscoelastic damping, Quart. Appl. Math, to appear.

[37] G.P. Menzala, E. Zuazua: Explicit exponential decay rates for solutions of von Kármán's system of thermoelastic plates, C. R. Acad. Sci. Paris Sér. I Math., 324, $1997,49-54$.

[38] G.P. Menzala, E. Zuazua: Energy decay rates for the Von Kármán system of thermoelastic plates, Adv. Differential Equations, to appear.

[39] C.S. Morawetz: Exponential decay of solutions of the wave equation, Comm. Pure Appl. Math., 19, 1966, 439-444.

[40] K. Narukawa: Boundary value control of thermoelastic systems, Hiroshima Math. J., 13, 1983, 227-272.

[41] A. Pazy: Semigroup of linear operators and applications to partial differential equations, Springer-Verlag, New York, 1983.

[42] D.C. Pereira, G.P. Menzala: Exponential decay of solutions to a coupled system of equations of linear thermoelasticity, Comput. Appl. Math., 8, 1989, 193-204.

[43] J.E.M. Rivera: Decomposition of the displacement vector field and decay rates in linear thermoelasticity, SIAM J. Math. Anal., 24, 1993, 390-406.

[44] J.E.M. Rivera, R. Racke: Smoothing properties, decay and global existence of solutions to nonlinear coupled systems of thermoelastic type, SBF series No. 287, Bonn University, 1993.

[45] D.L. Russell: Exact boundary value controlability theorems for wave and heat processes in star-complemented regions, in Differential games and control theory, Roxin, Liu, and Sternberg, Eds., Marcel Dekker Inc., New York, 1974, 291-319.

[46] D.L. Russell: Decay rates for weakly damped systems in Hilbert space obtained with control-theoretic methods, J. Differential Equations, 19, 1975, 344-370.

[47] L. De Teresa, E. Zuazua: Controllability of the linear system of thermoelastic plates, Adv. Differential Equations, 1, 3, 1996, 369-402.

[48] E. Zuazua: Controllability of the linear system of thermoelasticity, J. Math. Pures. Appl., 74, 1995, 291-315. 